\title{
-broich. Anmerkungen zu einer Landschaft Oswald Eggers
}

1

»Natur als Landschaft«, so Joachim Ritter in seinem grundlegenden Aufsatz »Landschaft", »ist Frucht und Erzeugnis des theoretischen Geistes" (Ritter 1974, 146). Theorie, verstanden im weitesten Sinn als Betrachtung (theoria), ist auch für Oswald Egger ein zentrales Element seiner Dichtung. Reflektierende Darstellungen von Landschaften tauchen in fast allen seiner Texte an zentraler Stelle auf. Zu nennen wären etwa der »Brachacker« (Egger 1999) in Herde der Rede, die »arealen Areale« (Egger 2001) in Nichts, das ist, die »Feld- und Fleckenlandschaft« (Egger 2010) in Die ganze Zeit oder das Val di Non (2017). Im Folgenden soll es um die Wahrnehmung der Landschaft im 2003 erschienenen Band -broich. Homotopien eines Gedichts gehen. -broich entfaltet eine Literatur der Landschaft des Niederrheins, wie sie etwa auch im Werk Jürgen Beckers zur Sprache kommt. Von den frühen Texten Felder (1964) und Umgebungen (1970) bis hin zum Gedichtband Aus der Kölner Bucht (2009) versucht Becker immer wieder, die sogenannte Kölner Bucht als Lokal des Schreibens darzustellen. In den »Vorbemerkungen« zu Aus der Kölner Bucht heißt es: »In den Gedichten dieses Bandes spricht die Kölner Bucht mit, kommt ihr Monströses, ihre beschädigte Schönheit, ihr Reichtum an Widersprüchen, an Bildern und gleichbleibenden Geräuschen, eine nicht nachlassende Faszination zu Wort.« (Becker 2009, 10) Landschaft ist für Becker »kein poetisches Thema« im klassischen Sinn, sondern »Material, [...] etwas Verborgenes, Nochnichtentdecktes, etwas Sichveränderndes, ein[] Rest, ein[] riesige[r] Rest von nichterzählter Geschichte« (Becker 2009, 10). Auch in Eggers -broich spricht die Landschaft der Kölner Bucht im Sinne Beckers mit. Dabei bildet Egger Landschaft nicht einfach ab oder erschöpft sich in Beschreibungen, sondern erkennt in dieser ein Reservoir an sprachlichem Material. Dieses widersetzt sich der geläufigen Sprache der Landschaftsbeschreibung; es lässt diese zuweilen monströs, beschädigt, widersprüchlich erscheinen. Die Schönheit der Kölner Bucht ist deformiert, sie zeigt sich im ruinösen Abbruch der Sprache.

Der Grund des Wortes -broich und damit des Titels von Eggers Text ist ein Bruch. Orte, deren Namen ihren Grund im -broich haben, verweisen auf eine Bruch- oder Sumpflandschaft. So heißt es im sprachwissenschaftlichen Wörter-

Jörg Kreienbrock, Evanston 
buch Die Bonner Flurnamen lapidar: »Broich, Bruch = sumpfiges Gelände« (Dietz 1973, 110). Folgt man Grimms Wörterbuch, bezeichnet der Bruch einen »feuchten wiesengrund, der beweidet und betreten werden kann« bzw. eine »dem strom durch dämme abgewonnene niederung, sumpfgegend « (Grimm 1854-1961, Bd. 2, Sp. 410). Ein Broich stellt somit ein Gebiet auf der Schwelle von Land und Fluss, Festem und Flüssigem ${ }^{1}$ dar. Diese eigentümlich grundlose Landschaft beschreibt Egger in -broich als aus »Bruchgefilden Unfeldern« (Egger 2001, 16) bestehend: »Nach der Tiefe stehender Wasser wird selten gefragt, vielleicht, weil man ohnedies schon weiß, dass ihr Grund mehrenteils aus Quellen, Triebsand, Sumpf, Morast, Schlamm, aber selten von Steinen, Wak und Kiesel durchsetzt besteht - daher immer tief ist und folglich oder nicht leicht durchwatet werden kann." (Egger 2001, 16) Das, was sich in -broich zeigt, ist keine unberührte Natur, sondern das Resultat kultureller Überformung. Es sind Dämme, die den Strom eingrenzen, seinen kontinuierlichen Fluss in feste, definierte Bahnen leiten, und dadurch brüchigen Boden $\mathrm{zu}$ Tage treten lassen. Zwischen Natur und Kultur entfaltet sich in -broich eine brüchige Sprachlandschaft grundloser Namen und Örter. Statt in fester Erde zu wurzeln, besteht sie aus einem semantischen Geflecht aus Zitaten, Anspielungen, Referenzen.

In diesem Sinn muss der Bruch im poetischen Werk Eggers als Teilung, Spaltung, Unterscheidung verstanden werden. Sprache ist gebrochen, uneins. Dieser Bruch der Sprache deutet sich bereits in der Schreibweise des Titels -broich an. Zum einen verweist der Bindestrich auf einen Wörterbucheintrag, wie er sich etwa in Grimms Wörterbuch finden lässt. Andererseits markiert er das Wort als ab- und aufgebrochen, fragmentiert. Das, was den -broich zu einem Ortsnamen vervollständigen würde, fehlt. Dem Bruch fehlt die Ergänzung zur Fülle; diese zeigt sich nur als Leere, fehlendes Glied des Ortsnamens. Das Ortnamengrundwort als Wortstamm bedarf der Vervollständigung durch eine Vorsilbe, um einen konkreten Ort zu bezeichnen. So könnte etwa der -broich durch die Hinzufügung der Silbe Hom zu Hombroich, dem Wohnort Eggers in der nördlichen Kölner Bucht, erweitert werden. Andere lokale Ortsnamen sind zum Beispiel Grevenbroich, Korschenbroich oder Kleinenbroich. Es ist jedoch auffällig, dass die Vervollständigung des gebrochenen Namens -broich zum konkreten Ort ausbleibt. Der Wohnort ist ohne festen Grund, auf dem der Dichter Fuß fassen könnte: »Zum einen diese Literde der abdacht schwarzgepflügten Äcker, immernass. Und

1 Vgl. Hunold 2016, 356: „Das deutsche Wort Broich kann abgeleitet werden von vaskonisch berr-ager $=$ wiederauftauchen und oih-an $=$ Wald, Busch, wobei die jeweils ersten Silben zur Bildung des Grundwortes benutzt werden. Eine andere Deutung ist ebenso möglich: bro = borbor $=$ brodeln, sprudeln, und ihi $=$ Binse. Daneben ist aber auch eine Deutung auf keltischer Grundlage möglich. Urk. Vroiko-s = Heidekraut, Heide, irisch froesch, Gen. froich > broich.«. 
doch, noch überzog sie Sommern eine krustharte Risshaut, so dass Schär und Rillfse trockenschroff scheinten, und ich sinke bis zu den Knien in diesen dumpfen, Torfen Ungrund. (Egger 2001, 16) Was trittfest erscheint, ist in Wirklichkeit brüchig. Dies gilt auch für das Ortsnamengrundwort -broich. Es bezeichnet weder den Grund des Ortes noch des Namens, sondern unter seiner rissigen Oberfläche öffnet sich ein Ungrund. Eine Gegend, die sich nicht zum Betreten, Wohnen, Wurzeln schlagen eignet.

\section{2}

Die gebrochene Landschaft, die in -broich auftaucht, entzieht sich zwar der genauen Bestimmung, ist aber dennoch in Teilen lokalisierbar. So nennt Egger mehrmals den Jaspersberg und die Halde Anna in der Nähe der Stadt Alsdorf im Westen der Kölner Bucht. Diese liegen in der Nähe des Broichbachtals, dem Ort Broich und dem Naherholungsgebiet Broicher Weiher. Als Teil eines ehemaligen Steinkohlereviers trägt diese gebrochene Landschaft die Zeichen menschlicher Bearbeitung, industrieller und landwirtschaftlicher Nutzung. Was sich in -broich zeigt, ist eine »Ruderalflora« (Egger 2001, 71). Der Begriff >Ruderalvegetation bzw. Schuttvegetation, der seit dem 18. Jahrhundert in der Botanik verwendet wird, bezeichnet »die vorwiegend krautige Vegetation anthropogen stark veränderter und/oder gestörter Wuchsplätze, sofern diese weder land- noch forstwirtschaftlich genutzt werden« (Brandes und Giese 1991, o. S.). Sie »besiedelt offene und häufig gestörte Flächen der Siedlungen, Industrie- und Entsorgungsanlagen sowie Verkehrswege. Sie ist auf kleinstem Raum sehr vielfältig, da bereits geringe Änderungen einzelner Standortfaktoren zu quantitativen oder qualitativen Veränderungen in der Artenzusammensetzung führen. Die Ruderalvegetation ist zudem sehr stark von historischen Faktoren geprägt; sie widerspiegelt geradezu unsere Kulturgeschichte« (Brandes und Giese 1991, o. S.) Diese Definition des Pflanzenbiologen Dietmar Brandes macht deutlich, dass Eggers Dichtung des -broich sich dort ansiedelt, wo Natur und Kultur ineinander übergehen. „Instruktiv«, so heißt es im Eintrag vom 16. Juli, »ist die noch in die Rede entstehende Ruderalflora, Trümmerpflanzen, die sich um distrikt zerstörte Flurruinen ausgebreitet haben« (Egger 2001, 71). Eggers Landschaftsdichtung wächst gleichsam wie Trümmerpflanzen auf Ruinen. Sie gründet auf den von der Industrie zerstörten Brachen des rheinischen Bergbaus und blüht dort, wo lebendige Natur abgestorben ist. So wie die Ruderalflora sich auf den Trümmern der Kulturlandschaft ausbreitet, so siedelt Eggers Rede auf Ruinen, den Nachlassenschaften einer zerfallenen Ordnung der Sprache. Der prosaische Grund der Sprache ist ruiniert aber gerade dadurch offen 
für neue Ansiedlungen, offen für neue, poetische Gesellschaften von Pflanzen und Wörtern. Ruderale Standorte bilden einen fruchtbaren Boden für fremde Arten, die aus anderen Lebensräumen einwandern und die Trümmer des Eigenen belebend verwandeln.

Die Bergehalde Jaspersberg, die aus Abraummaterial des Steinkohlebergbaus besteht, ist paradigmatisch für einen verwüsteten Ort, der langsam kultiviert wird. Eggers Sprache der Flora und Fauna siedelt auf Materialhalden, d. h. auf Schutt und Trümmern, in Gegenden, wo die Natur ge- wenn nicht zerstört wurde. Landschaft kann für Egger in -broich daher nicht zu einem Symbol einer harmonischen Vermittlung von Subjekt und Objekt werden. Eggers Dichtung als Rekultivation versucht weder, die Schäden der Zivilisation elegisch $\mathrm{zu}$ beklagen, noch diese in einer wiederzugewinnenden Idylle zu überwinden. Eggers Eintrag vom 23. Juli zum Jaspersberg zitiert in diesem Sinne einen Bericht des Naturkundlers Wolfgang Voigt, der auf der Internet-Seite des Naturschutzbundes Aachen-Land veröffentlicht ist. Dort wird unter dem Titel »Bergehalden im Aachener Revier - eine Zukunft für die Natur « die Bergehaldenlandschaft im Aachener Revier beschrieben und Möglichkeiten der Renaturierung verschiedener Halden diskutiert. Seit 1986 gilt der Jaspersberg als sogenannter »Geschützter Landschaftsbestandteil«, d. h. er ist »ruhend, endgestaltet, rekultiviert« (Voigt 2003, o. S.). So wie die Halden der Landschaft um Aachen aus dem Material des Bergbaus bestehen, so ist Eggers rekultivierende Landschaftsbeschreibung aus dem Haldenmaterial von ihm gelesener Texte aufgebaut. Egger montiert zum Beispiel aus dem Material botanischer und zoologischer Fachsprachen neue Namen, die er mit geläufigen Bezeichnungen vermengt. In der Beschreibung »mehrere[r] Haldenkomplexe aus Schlammweihern« (Egger 2001, 70) am 15. Juli nennt Egger zum Beispiel die real vorkommenden Vogelarten Eisvogel, Feldschwirl und Krickente, aber auch imaginäre Spezies wie Rotsprenkel, Blässsäbel oder Deichralle. Diese Namen sind jedoch keine freien Erfindungen, sondern variieren oftmals Bezeichnungen, die Egger im Abschnitt über die Bergehalde Anna in Voigts Aufsatz aufliest. Dort heißt es etwa an einer Stelle: »Man kann [...] auf den Schlammflächen Bekassine, Bruchwasserläufer, Flußuferläufer, Rotschenkel und Waldwasserläufer entdecken [...]. Auf dem freien Wasser sieht man Stock- und Krickente sowie Bläß- und Teichralle« (Voigt 2003, o. S.). Eggers Poesie siedelt neue Wörter auf den Abraumhalden der Botanik und Zoologie an. Aus der Teichralle wird die Deichralle, aus der Bläßralle ein Blässsäbel. -broich ist ein Text sowohl exakter wissenschaftlicher Fachsprachen als auch poetischer Imagination. So wie die Landschaft die Spuren ihrer industriellen Vergangenheit trägt, ist Eggers Dichtung von entstellten Zitaten, gebrochenen Namen gezeichnet.

Das Verschleifen von Fremdem und Eigenem, Zitat und Erfindung setzt sich im Eintrag vom 24. Juli fort, welcher mit einem Zitat aus einem 1908 veröffent- 
lichten naturkundlichen Führer durch das Broichtal beginnt. In Peter Schiffers Führer durch das Broichtal heißt es: "Gleich einem großen, strahlenden Auge, das zum Himmel aufblickt, erscheint die glänzende Wasserfläche in ihrer grünen Umgebung. « (Schiffer 1908, 18) ${ }^{2}$ Egger zitiert diesen Satz, um den Eindruck der grünen Umgebung weiter auszudifferenzieren und $\mathrm{zu}$ verformen: »Gleich einem mächtig strahlenden Auge, das zum Himmel aufblickt, erscheint immens diese glänzende Hochmoor-Wasserfläche in ihrer grün-rings verheideten Umgebung in Mondflockmaaren, Gipskeupern und Löß zu Truppenübungsplätzen, eingewandelt in Versaumungsbereichen, quirlaktive Gilden, und Gewässersandarten auch. (Egger 2001, 79) Weiter unten im Text taucht erneut ein leicht abgewandeltes Zitat aus dem Broichtal-Führer auf. Aus Schiffers Satz »An der einen Seite rechts führt ein Weg, getrennt durch einen schmalen Uferstreifen, der mit Baumund Strauchwerk und verschlungenem Brombeergerank bewachsen ist, am Weiher vorbei. Wir wandern zum entgegengesetzten Ufer.« (Schiffer 1908, 18) wird in Eggers Umschrift: »An der einen Seite rechts führt ein Weg, getrennt durch Uferstreifen, die mit Baum- und Strauchwerks verschlingenden Brombeer räkelgert überbewachsen sind (am Tauchblatt-Weiher vorbei). Ich wandere zum wiederholt entgegengesetzten Ufer.« (Egger 2001, 79) Egger steigert die syntaktische wie semantische Komplexität des Zitats. Das Verschlungene des Wegs wird nicht nur wie bei Schiffer genannt, sondern spiegelt sich in der Syntax der Sätze wider. Eggers Sprache ist an dieser Stelle - wie der Weg um den Teich - verschlungen. Die einfache Beschreibung des naturkundlichen Führers durch das Broichtal entfaltet sich in eine Abfolge schillernd-glänzender Wörter. Schiffers Prosa vervielfältigt sich in Eggers Text zu immer neuen Reihen von Fachtermini, Neologismen und Adjektiven. Diese gleichen »selbstwendend mäandrierenden Fließgewässern mit Feuchthaldewäldern « (Egger 2001, 79). In einer Parenthese heißt es beispielsweise: »(Ockerquecken, Pest-Asche in den Salzgrashalden Tunkgelben Nelkenflecken Labsaat der Rohboden-Moränen Planierverstufungen und Kies-Bergesteppen, Kupferglanzgräser mit Striemendwurzeln Heck-Zacklinien und Füllwinkeln in den Leuchtwiesen Wasserschwaden Gips-Sprießeln Torfstichangern aber, Nektarquellen, wo Raupen, die sich in Knospen eingebohrt haben, mitunter unverblühten Pappelkätzchen zu Boden fielen)« (Egger 2001, 79). Aus den Vokabeln der Prosa der Naturkunde (ver-)formt Egger einen Text, der aus der Geradlinigkeit des Führers ausbricht, und eine andere, poetische Beschreibung der Landschaft imaginiert.

2 Eine andere Quelle für Eggers Beschreibung des Broichs stellt der Bericht »Natur in Alsdorf ein naturkundlicher Spaziergang « des Gymnasiallehrers und Naturkundlers Wolfgang Voigt dar; vgl. Voigt 2003. 


\section{3}

Die Ausstellung des Brüchigen im -broich, verstanden als Ungrund von Ort und Name, steht in eigentümlichem Widerspruch zum Untertitel des Bandes, Homotopien eines Gedichts, welcher dessen poetische Vorgehensweise mit dem mathematisch-topologischen Begriff der Homotopie zu fassen sucht. Der Begriff der Homotopie bezeichnet in der Mathematik die stetige Veränderung einer Gestalt. Homotopie ist der bruchlose Übergang einer Form in die andere. Eggers brüchige Landschaftsdichtung, so könnte man dementsprechend argumentieren, stellt einen Prozess der stetigen Deformation, einer kontinuierlichen Veränderung von Gestalten dar. Sie erschließt »folglich einen ganz anderen Wissensraum, weil sie Dinge ins Verhältnis setzt, die man im geometrischen Normalraum nicht entdecken würde« (Simons 2015, 278), wie es Oliver Simons mit Blick auf das Denken des Raums in der Topologie feststellt. ${ }^{3}$ Als Homotopien eines Gedichts stellt sie eine Literatur der Lagebeziehungen, Transformationen und Verfremdungen dar.

Die einundachtzig Vignetten, die den Band -broich ausmachen, stellen also, folgt man der topologischen Definition, einen kontinuierlichen Zusammenhang dar. Trotz dieser Stetigkeit sind Eggers Aufzeichnungen streng nach Tagen differenziert. Der Zeitraum vom 7. Mai bis zum 26. Juli stellt ein gebrochenes Kontinuum dar, oder, wie es Egger in einem anderen Zusammenhang formuliert, eine Diskrete Stetigkeit (Egger 2008). Die Homotopien des einen Gedichts vollziehen sich an vielen Tagen. Sie sind also gerade nicht stetig, sondern diskret, diskontinuierlich. Darüber hinaus ist der Text, wie viele von Eggers Dichtungen, von Zeichnungen, Skizzen und Fotos unterbrochen. Im Eintrag vom 12. Mai heißt es dementsprechend: »[I]ch will's mir denken, dieses andauernde Gefühl für Zeit gewinnen, dann jenen Raum einzunehmen, Bresche $\mathrm{zu}$ tun, in welcher unverstreichende Minute, die Sekunden sind, im Nu das stummbe Zwischenspiel von Einfalls-Bildern« (Egger 2001, 6). Die Daten sind Schnitte durchs Kontinuum der Zeit, sie sind wie Breschen oder Stanzen. Das Tagebuch -broich entfaltet sich in Form der Homotopien eines Gedichts. Dieses eine Gedicht als geformte Gestalt ist dem Leser jedoch entzogen. Es existiert nur im Plural, in der Form von Homotopien, von Transformationen, Verzerrungen. Es ist nicht unmittelbar lesbar, sondern stellt den prosaischen Hintergrund dar, vor dem sich die verschiedenen Tageseinträge als Homotopien des einen sich entziehenden Gedichts abheben. Die Reihe der Tage bildet ein Gitter, zwischen dessen Stäben - den einzelnen Ein-

3 Zur Bedeutung der Topologie für die Kulturwissenschaften vgl. Günzel 2007. 
trägen - sich Dichtung im Entzug zeigt. Das Gedicht ist das Changieren zwischen Figur und Grund, Präsentation und Privation, Poesie und Prosa.

Eggers Begriff der Homotopie kann in diesem Sinne als ironische Replik auf den von Michel Foucault entwickelten Begriff der Heterotopie gelesen werden. In seinem Aufsatz »Andere Räume« diagnostiziert Foucault: »Wir sind in der Epoche des Simultanen, wir sind in der Epoche der Juxtaposition, in der Epoche des Nahen und des Fernen, des Nebeneinander, des Auseinander. Wir sind, glaube ich, in einem Moment, wo sich die Welt weniger als ein großes sich durch die Zeit entwickelndes Leben erfährt, sondern eher als ein Netz, das seine Punkte verknüpft und sein Gewirr durchkreuzt.« (Foucault 1992, 34) Foucaults Rede von der Verknüpfung von Punkten formuliert, was in der Topologie Verbindbarkeit bzw. Zusammenhang heißt. „Ein Raum heißt wegweise zusammenhängend «, so der Mathematiker Tammo tom Dieck, »wenn je zwei seiner Punkte verbindbar sind « (tom Dieck 2000, 6). Foucault notiert: »Die Lagerung oder Platzierung wird durch die Nachbarschaftsbeziehungen zwischen Punkten oder Elementen definiert, formal kann man sie als Reihen, Bäume, Gitter beschreiben.« (Foucault 1992, 36) Zu den Transformationen topologischer Räume gehören das Dehnen, Stauchen, Verbiegen und Verzerren von geometrischen Figuren. Dieser Zusammenhang des Raumes, der sich durch die Verknüpfbarkeit seiner Punkte als Nachbarschaftsbeziehung ausweist, kommt in -broich sprachlich zum Ausdruck. Eine solche bei Egger oft vorkommende deformierende Veränderung der Wörter ist das Anagramm, wie es etwa in Herde der Rede als stetige Transformation von Herde, Erde und Rede auftaucht. Zwar verändert sich in der anagrammatischen Veränderung eines Wortes dessen äußere Gestalt, doch bleibt seine Struktur in dieser poetisch-topologischen Abbildung erhalten. Egger schreibt in Harlekinsmäntel \& andere Bewandtnisse mit Bezug auf Leibniz, einen der Begründer der Topologie: „In immer neuer Gestalt erscheinen die trotz stetiger Transformationen ständig dieselben bleibenden, unzerstörbaren Substanzen.« (Egger 2017, 77) Trotz vielfältiger Deformationen, d.h. immer neuer Gestaltwerdung bleiben Äquivalenzen zwischen Texten, Wörtern, Buchstaben bestehen.

Dieses Modell von Trans- bzw. Deformationen einzelner sprachlicher Elemente lässt sich auf die spezifische Intertextualität von Eggers Dichtung übertragen. Das Zitieren von Angelesenem stellt stetige Zusammenhänge zwischen verschiedenen Texten, Diskursen, Kulturen dar. Dabei deformiert Egger im Vorgang der Wiedergabe das Bestehende, ohne seine materielle Substanz zu zerstören. Nur aus den Örtern der Überlieferung kann Dichtung »in immer neuer Gestalt« entstehen. Eggers Schreiben entfaltet sich in der Spannung einer »Topik der geschlossenen Räume und der Hyperbolik der offenen Horizonte« (Haverkamp 1998, 268), wie es Anselm Haverkamp in einem anderen Zusammenhang formuliert. In »Über die natürliche Magie der Einbildungskraft. Ein Briefgespräch in zehn Kapiteln mit 
Michael Braun« entwickelt Egger seinen Umgang mit der literarischen Tradition als Toposforschung entsprechend als einen »Wegzusammenhang«: »Wenn man sich in den Wegzusammenhang einer Überlieferung stellt, bleibt es, denke ich, ungleich wichtiger, wozu und wohin man das Übernommene führt und führen wird (als beispielsweise aus welchem Grund man es erschlossen oder überhaupt vernommen habe).«(Egger 2000, o. S.) ${ }^{4}$ Intertextualität entsteht für Egger durch die Bewegung durch eine historisch überformte »Fundlandschaft« (Egger 2000, o. S.). Diese zeigt sich niemals in ihrer ursprünglich-natürlichen Unberührtheit, sondern ist immer schon kulturell überformt. Es geht Egger nicht um die Erhaltung oder Weiterschreibung von Tradition als Nachdichtung oder Übersetzung, sondern darum, "neue Ungründe zu erfinden in einer Fundlandschaft" (Egger 2000, o. S.). Diese ist im Sinne von Jean-Luc Nancy eine radikal entwurzelnde. ${ }^{5}$ Was von Egger in brüchigen Textlandschaften und aufgelesen und übernommen wird, ist dem Grund entzogen. Seine Gedichte entgründen.

\section{4}

Eggers Broich-Landschaft erscheint wie durch ein Gitter. Paradigmatisch für diese Rasterung des Blickfelds ist der Eintrag vom 25. Juni:

Das Abendlicht flutet in konturen, goldroten Bahnen durch die Halme, Fugengras glimmt leuchtorange, wie Linien im zerschlissenen und arg alten Samt. Verkappte Perspektiven umschließt der Einblick in diese Szenerie. Suchen, tasten, inne sein, von einem Mal zum anderen verkapselte Ostritz-Hüllen, Hilbe, die wie vorsortierte Kumpf-Muffe ausgebreitet scheinten im Areal der Geländebrachen Unfelder. Vorn zeigt sich die Wiese halden (unberührt, aber gangbar), Schmielen und ihre Ähren Prärealien stehen in wattiert-Kissen Büscheln, nach wenigen Schritten schiebt sich die Reihenweise ineinander, wird gittrig und zersplitterte schraffur zu Paletten und Senkrecht-Schatten. - Aber das Augenlicht dringt noch und noch nächtiger in die vertiefte Weise, stößt erst völlig und hinten, aber wo? Auf opakte Wallmgatter aus Zwerch- und Senkrechtlatten der Tuilerie, Zingel aus Altziegeln, die sich verschanzten und Zäune vermauerten statt einseitwendiger verunwinden. Durch Haufenwolken hindurch, Spalt-breit einer Lücke, senkt sich die Sonne untergehend um den Horizont und berührt jetzt die Zaunlatten des Rastergartens.

(Egger 2001, 50)

Diese Passage fragt nach der spezifischen Räumlichkeit einer durch das konture Abendlicht gerastert erscheinenden Landschaft. Sieht man im Blick durch die Gitterstäbe Figur oder Hintergrund, Dinge oder Schatten? Manfred Smuda

4 Zur Intertextualität Eggers vgl. Prammer 2014; De Felip 2011.

5 Vgl. Nancy 2006. 
betont dementsprechend in einem Aufsatz über Natur als ästhetischem Gegenstand die Notwendigkeit, im Prozess der perzeptiven Konstitution von Landschaft zwischen Figur und Grund zu unterscheiden: »Eine Spezifizierung erfährt das GestaltPhänomen in der `Figur-Grund «-Relation, deren wichtige Funktion für das Sehen von Landschaft wohl kaum übersehen werden kann. Wenn ich wechselweise die umschlossene Fläche als Figur und die umschließende als Grund oder die Fläche, die vorher als Figur erschien, als Grund sehen kann, so dokumentiert auch dieser Sachverhalt den perzeptiven Charakter des Landschaftsbildes.« (Smuda 1986, 55)

Das Wechseln der Perspektive zwischen Figur und Grund ist es, die den Raum der Landschaft etabliert. In diesem wird es möglich, Gegenstände zu erkennen, indem sie sich deutlich von ihrem jeweiligen Hintergrund absetzen. »Landschaft konstituiert sich uns also«, so Smuda, »indem sie sich als `Gestalt» von der uns umgebenden Natur abhebt, indem sie sich gegen dieses >Herum behauptet. Sie kann sich nur gegen es behaupten, indem sie zugleich in dieses >Herum` eingebettet bleibt, darauf wartend, daß wir in der Konfiguration des Mannigfaltigen eine Sinneinheit erfassen und sie innerhalb des Ganzen der Naturwirklichkeit und zugleich gegen es, bzw. auf seinem Hintergrund, als >Gestalt « etablieren.« (Smuda 1986, 49) In Diskrete Stetigkeit diskutiert Egger die Frage nach der Beziehung von Figur und Grund in der Landschaftsdarstellung anhand von einem »Bruchwald « (Egger 2008, 50). In dieser Umgebung nahe dem Broich vollzieht Egger ein wahrnehmungspsychologisches Element, indem er seinen Blick zwischen den Bäumen wandern lässt:

Lasse ich nun aber >den Blick zwischen zwei in verschiedener Entfernung befindlichen, jedoch benachbarten Stämmen willkürlich hin- und herwandern, so werde ich im Zwischenraum zwischen diesen ১beiden Bäumen` im eigentlichen Sinne gar nichts sehen. Obwohl ich nun zwischen >diesen beiden` Stämmen, und ebenso zwischen den Stämmen, welche sich näher befinden, im engeren Sinn gar nichts sehe, so erscheint doch Nebel zwischen den Stämmen, welche weiter verwanderten als die, Schwaden wie Baumstämme im Schnee, zwischen denen ich stapfend vorging, deutlich sraumgreifendes Grau .

(Egger 2008, 51)

Jeder Wald verwandelt sich durch den wandernden Blick in einen Bruchwald. Als »Wand« oder »grüne Masse« (Egger 2008, 50) ${ }^{6}$ ist er durchsetzt, gleicht er einem Gitter, in dem sich im Widerspiel von Figur und Grund neue Perspektiven eröffnen. Laut Grimms Wörterbuch bezeichnet Gitter »ein feineres, künstlich gearbeitetes, ebenes gefüge aus dünnen parallel, kreuzweise oder verschränkt gestellten stäben (namentlich metallenen), oder netzartig geflochtenen drähten oder durchbrochenen figuren« (Grimm 1854-1961, Bd. 7, Sp. 7572). Zwischen dem Git-

6 Zum eigentümlichen Begriff der Waldwand vgl. Donhauser 2016. 
ter der parallelen Stämme erscheint der Grund des Waldes auf der Schwelle zur Figuralität in »durchbrochene[n] figur[en]«. Im »Verwandern« - ein Neologismus, der sowohl an eine wandernde Bewegung als auch die verändernde Beobachtung erinnert - springt der Blick des Dichters zwischen Figur und Grund. So entsteht im Bruch eine Landschaft. Die Broich-Landschaft erschließt sich im kontinuierlichen Wechsel der Perspektive, die - immer auf dem Sprung - zwischen Figur und Grund, Lyrik und Prosa oszilliert. Egger fährt fort: »Im Wald von Gegenständen, der mich umgibt, ein gleichbleibender Dichtwuchs von Bäumen, finde ich mich nur dadurch zurecht und werde über das Durcheinander der auf mich eindringenden Gegenwarten gewahr, daß ich Gegenstände von andern trenne, daß ich ihnen Umriß, Maß und Gestalt gebe, mithin im Mannigfaltigen Eins um Eins absondere« (Egger 2008, 141). Vor dem Hintergrund des Walds zeichnen sich einzelne, diskrete Formen ab. Der »gleichbleibende[] Dichtwuchs von Bäumen« wird zu einem Gitter einzelner Stämme und Stäbe. Man sieht entweder den Wald oder die Bäume, nie aber den Wald und die Bäume. Das Oxymoron der diskreten Stetigkeit kann in diesem Sinn als eine Meditation über das von Christoph Martin Wieland eingeführte Sprichwort, dass man den Wald vor lauter Bäumen nicht sehen könne, verstehen. Man sieht entweder diskrete Formen oder den kontinuierlich zusammenhängenden Grund, nicht aber beides gleichzeitig. Richtet sich das Augenmerk auf den einzelnen Baum, so entzieht sich der Wald; rückt dieser in den Fokus, so gehen die Bäume der Beobachtung verloren. Der abgebrochene Wortteil -broich bezeichnet diesen Punkt des Umschlags von Teil zu Ganzem, von Landschaft zu Natur, Figur und Grund. Es ist der Bruch, der immer nur im Umschlag des Augenmerks aufblitzt. Er hat keine räumliche Ausdehnung oder zeitliche Dauer, sondern konstituiert diese als deren Ungrund.

Mit dem Wort Tuilerie im Eintrag vom 25. Juni erinnert Egger nicht nur an den paradigmatischen »Rastergarten « überhaupt: den Jardin de Tuileries in Paris, sondern verweist ebenfalls auf eine Ziegelei. So verwandelt sich eine Ziegelei, Artefakt der rheinischen Industrielandschaft, zum französischen Jardin de Tuileries. Das Areal der Geländebrachen Unfelder wird zu einem Rastergarten. Die Landschaft, die durch das Abendlicht konturiert wird, erinnert somit zugleich an die strenge Geometrie barocker Gärten als auch an die industrielle Vergangenheit der Landschaft, welche nur noch als Industriebrache wahrgenommen wird. Die strenge Geometrie barocker Gartenbaukunst zersplittert zum Brachland. Natur erscheint nur mehr als Broich, brach liegend. Das Wort Zingel, nah dem in der Ziegelei hergestellten Ziegel, bezeichnet laut Grimms Wörterbuch den »befestigungsgürtel einer stadt« als auch »streifen, bänder an felsbergen« (Grimm 1854-1961, Bd. 31, Sp. 1390). Erneut taucht ein Wort in Eggers Beschreibung auf, das sowohl Natur als auch Kultur zuzuordnen ist. Die befestigte Grenze einer Stadt wird durch einen Streifen bzw. einen Gürtel (von lat. cingulum) markiert, der ursprünglich 
eine Palisade darstellte, d. h. eine Verschanzung durch Pfähle. Dieses Pfahlwerk, so Eggers Beschreibung der rheinischen Tuilerie, wurde durch die Befestigung mit Ziegeln zur Zingelmauer. Doch in der Ruinierung dieses Baus, der Verwüstung zur Brache öffnen sich neue, zuvor »verkappte Perspektiven«, »Einblick[e] in diese Szenerie« (Egger 2001, 50), die jedoch nicht eindeutig zwischen Natur und Kultur, Ziegelei und Wiese, Vorder- und Hintergrund unterscheiden, sondern Landschaft »gittrig und zersplittert« erscheinen lässt.

In diesem schraffierten Bildfeld trifft der Blick auf ein »Wallmgatter aus Zwerch- und Senkrechtlatten«. Bei Grimm heißt es: "gatter ist wesentlich ein aus stäben, latten $u$. ä. hergestellter verschlusz mit lücken, im unterschiede von thür und thor, die sich aus anschlieszenden oder eingefugten brettern bestehn, wie die stäbe stehn, ob neben einander oder gekreuzt, oder über einander liegend, ist dem begriffe jetzt gleichgültig, doch fehlt wenigstens eine kreuzende latte fast nie; im 13. jahrh. aber wird das kreuzen, verschränken auch als dem gater wesentlich gedacht« (Grimm 1854-1961, Bd. 4, Sp. 1504f.). Auf ähnliche Weise wie ein Gatter kreuzt und verschränkt Egger die Wörter. Der Text wird bei ihm zu einem Gitter aus Buch-staben. Wesentlich ist das Nicht-Anschließende, Nicht-Eingefugte, Diskrete, welches Lücken lässt, die Kontinuität des Bilds unterbricht. Statt einer Mauer sieht Egger eine Palisade, oder - wie es Walter Benjamin mit Hinblick auf den Vorgang des Übersetzens formuliert - eine Arkade. »Die wahre Übersetzung ist durchscheinend, " so Benjamin in Die Aufgabe des Übersetzers, "sie verdeckt nicht das Original, steht ihm nicht im Licht, sondern läßt die reine Sprache, wie verstärkt durch ihr eigenes Medium, nur um so voller aufs Original fallen. Das vermag vor allem Wörtlichkeit in der Übertragung der Syntax und gerade sie erweist das Wort, nicht den Satz als Urelement des Übersetzers. Denn der Satz ist die Mauer vor der Sprache des Originals, Wörtlichkeit die Arkade« (Benjamin 1991, 18). Durch seine Brüchigkeit ist die Arkade wie die von Egger beschriebenen Gatter, Gitter, Palisaden und Zäune als »Instanz[] der Sonderung« (Hamacher 2004-2005, 141) keine Mauer, sondern verwandelt sich zu einer durchlässigen Zone. Sie öffnet den Blick für die Wörter in ihrer Buchstäblichkeit. Die gefugte Mauer des Satzes als Prosa wird zersetzt durch die lyrische Wörtlichkeit und die horizontal-syntagmatische Gliederung durch eine vertikal-paradigmatische Ordnung unterlaufen. Im Fall von -broich heißt das, dass in Eggers Dichtung Wörter verschlagen sind, die in ihrer Wörtlichkeit z. B. als Zitat oder Fremdwort auf einen anderen Grund von poetischer Sprache verweisen, der sich weder eindeutig der metonymischen Logik der Prosa noch der metaphorischen Ordnung der Lyrik zuordnen lässt. 


\section{5}

Paul Celans Gedicht "Sprachgitter «, auf das Egger an mehreren Stellen anspielt, beginnt mit der Evokation eines Augen-Blicks, der zwischen Stäben eine andere, freie Form der optischen Wahrnehmung imaginiert: »Augenrund zwischen den Stäben. / Flimmertier Lid / rudert nach oben, / gibt einen Blick frei.« (Celan 1986, 147) Dieses Freigeben des Blicks wird von Egger in verschiedenen, zwischen Phänomenologie und Wahrnehmungspsychologie schwankenden Experimenten erprobt. In Diskrete Stetigkeit gibt es zum Beispiel einen direkten Verweis auf stroboskopische Experimente: »Ich habe das Experiment mit einem sich drehenden Speichenrad [...] nachgestellt.« (Egger 2008, 121)

Im Eintrag vom 19. Juni heißt es:

In meinem Zimmer studiere ich ein Muster aus einheitlich verteilten Details (Tapete und Gardine), und zwar derart, dass sein begrenzender Rand außerhalb des Blickfelds bleibt. [...] Ich versuche, aus einiger Entfernung durch das Muster in die Tiefe zu blicken, mein Augenmerk auf immer weitere Entfernungen zu orientieren. So und sobald es mir gelungen ist, durch (und durch) das Schmuckmuster zu schauen, stellt sich eine überraschende Betrachtung ein: an der Stelle des ersten (ungewahr) realen Musters ergründe ich tiefer liegend gleich und gleich ein zweites (gewahr areales), mit akkurat aber ersichtlich größeren Abmaßen. Das erste, reale Muster ist verschwunden, und an dessen Stelle sehe ich eine wesentlich gläserne Wand.

(Egger 2001, 44)

Die Welt erscheint durch ein Raster. Fokussiert der Beobachter statt des vordergründigen Musters des Zimmers dessen Hintergrund, geht der Blick von der Oberfläche in die Tiefe, so entsteht eine neue, andere Strukturierung des Sehfeldes. Der Vordergrund tritt zurück aus der Wahrnehmung - er wird »ungewahr« - und an seine Stelle tritt der Hintergrund, der zwischen den Formen des Musters als diskrete Form zu Tage tritt. Egger weiter: »entweder man sieht die Gardine vor dem Fenster oder die Landschaft im Hintergrund." (Egger 2001, 44) Die Rahmung bzw. Rasterung des Blickfeldes zu einem derart arealen Areal geschieht durch das Fenster: »Auf dem Fußboden des Zimmers ist die helle, Viereck-Fläche des Fensters zu sehen, die wiederum durch den Fensterrahmen in kleinraute Karrees unterteilt ist.« (Egger 2001, 55) Der Blick durchs Fenster wird gleichzeitig gerahmt und geteilt. Das Blickfeld ist gerastert. Das Wort Raster, das aus dem lateinischen rastrum für Rechen, Hacke abgeleitet ist, deutet auf den Prozess der Kultivierung von Natur. Durch das Rastern entsteht Ordnung. Bernhard Siegert verweist in diesem Sinne auf die ambivalente Kulturtechnik des Rasters zwischen mimetischer Abbildung und projektiver Konstruktion: »Als Kulturtechnik spielt das Raster eine doppelte Rolle: Es ist ein diagrammatisches Bildgebungsverfahren, das nach dem Prinzip arbeitet, Daten unter bestimmten Adressen abzuspeichern, das heißt, es ist repräsentational und zielt darauf, eine hohe mimetische 
Evidenz zu erzeugen. Aber zugleich ist das Raster auch ein Realitätsgebungsverfahren, eine Technik, Welt als Welt von Objekten zu konstituieren, die von einem Subjekt vorgestellt werden, das heißt, es ist operativ, differenzbildend und zielt auf die Beherrschbarkeit des von ihm Erfassten. «(Siegert 2003, 93) Das Raster markiert entsprechend »den Ort der Differenzierung und Entdifferenzierung von Repräsentation und Operation« (Siegert 2003, 93).

In einem Gespräch vergleicht Egger diese gleichzeitig strukturierenden und entstrukturierenden Prozesse des Rasterns, die man mit Holger Steinmann als Grund einer »Sperrpoetologie« (Steinmann 2013, 287) ${ }^{7}$ deuten könnte, sowohl mit der Bewegung von Rilkes Panther hinter den Gitterstäben seines Käfigs als auch mit Moiré-Phänomenen auf Textilien:

Dies kommt zumindest dem recht nahe, was das Wort `Moiréı aussagt: es handelt sich ja nicht um das (unendlich) fortgesetzte Weben und Verflechten (von Gedanken und Worten) zum Textil (zum globalen, Welt-umspannenden zumal, das dem Erdkreis kongruent sein will), sondern meint das strikt lokale Phänomen gittriger, unruhiger Bildmusterung, das durch die Überlagerung zweier (Gewebe-) Strukturen entsteht und (auf Textilen) zumeist als >Wässerung، erscheint. Wenn man spalier an einem Zaungitter vorbeigeht, und es befindet sich, ein wenig versetzt, ein zweiter Gitterzaun dahinter, wird man dieses Phänomen ereignen sim Vorübergehn` (wie Rilkes >Panther`). Mithin entgeht man einer Welt, in der das Wahre das Gemachte war. Und stanzt und schlüpft in eine zweite Wirklichkeit der ersten (in den Gewahrsam des Gewahren).

(Egger 2003, o. S.)

In einem Textil-Fachwörterbuch wird das Moiré wie folgt beschrieben: «Moiréeffekt, entsteht durch Überlagerung zweier Gittersysteme (Interferenz).« (Kießling und Matthes 1993, 255) Es gibt entsprechend, so Egger im Eintrag vom 17. Juli, »in Rede entstehende[] Moiréarten« (Egger 2001, 72) bzw. eine Poesie »[n]ach dem Muster«, wie es Egger in einem Aufsatz über »symplektische[] Wortgeflechte« (Egger 2014, 285) ${ }^{8}$ bemerkt. Der Anthropologe Gregory Bateson beschreibt »Moiré Phenomena« folgendermaßen: »Interesting phenomena occur when two or more rhythmic patterns are combined, and these phenomena illustrate very aptly the enrichment of information that occurs when one description is combined with another. In the case of rhythmic patterns, the combination of two such patterns will generate a third.« (Bateson 1979, 79) Die Anreicherung von Information, von der Bateson spricht, ist das Ergebnis einer Überlagerung, Interferenz bestehender Muster, welche im Moment ihrer Überblendung etwas sichtbar werden lassen, welches sich der geläufigen Wahrnehmung entzieht. Auch in Diskrete Stetigkeit diskutiert Egger »Moiré-Erscheinungen« als Infrastruktur des Poetischen und stellt fest:

7 Vgl. auch Steinmann 2009.

8 Vgl. Endres 2014. 
»War ich erst auf Moirés aufmerksam geworden, traten diese mir bald auf Schritt und Tritt als häufige, zerstreute, gleichwohl geordnete Erscheinung entgegen" (Egger 2008, 122). Diese zeigen sich zum Beispiel als »Verschiebungen und Koinzidenzen zwischen Strich und Gitter wenig verschiedener Gitterkonstanten « (Egger 2008, 123). Und: »Das Moiré-Gitter bewegte sich wie ein Vorhang, der sich vor offnem Fenster hob.« (Egger 2008, 123)

Der Perspektivwechsel zwischen Vorder- und Hintergrund, der es erlaubt, das Blickfeld anders zu strukturieren, und die einzelnen Elemente der Landschaft neu zu sehen, findet auch auf sprachlicher Ebene statt. So wie der Blick das Wahrnehmungsfeld rastert und es durch die Verschiebung des Fokus bzw. der Rahmung zu neuen Bildern kommt, so selektiert der Dichter Wörter und stellt sie in neue Konstellationen. Am 7. Juli warnt Egger:

Ich sollte nicht leichtfertig neue Wörter schöpfen aus dem Pool und Pulver verunheimlichter Genügen, jedes Wort für Wort ist vorgenannt Diskretum, das (für sich) allein keine Sinne zur Bedeutung auslegte, aber völligt, und ganz teilhat am Wissen in liaison mit allenthalben anderen (und vorigen und solchen, die noch werden wollen und selbdritt vergegenwärtigen), die unverzehrte (nicht: verunzierte) Störflut von Ereignissen in taubfrischen Wörtern erschöpfte sich nach Strukturen Grundformen, diese tief-Genüge, in Empfindbilder getauchten Glieder und Gelenke im Zusammenhang, herauszuheben und in wie Siebende Reuse eingefacht und ins Trockendock verschoben aus seinem Element (gelüpft) zu tun.

(Egger 2001, 62)

Egger beschreibt hier den Vorgang der Dichtung als einen der Schöpfung. Diese produziert jedoch nichts Neues, sondern filtert Wörter aus der Flut der Sprache. Dichtung hebt Wörter als diskrete Einheiten aus dem geläufig-prosaischen $\mathrm{Zu}$ sammenhang heraus, um aus ihnen neue Verbindungen herzustellen. Dieser Prozess der Neugliederung von Sprache, welche die syntagmatischen und paradigmatischen Achsen zu einem Gitter verschränkt, gleicht der Rasterung der Landschaft durch den aufmerksamen Blick. Wörter entstehen im Heraussieben aus »Strukturen Grundformen«, der Verschiebung aus dem »Element« des »Pool[s]« in eine Ordnung des Rasters. Die Reuse ist das Werkzeug, mit dem diskrete Wörter aus dem Fluss der Rede herausgesiebt werden. Es werden keine neuen Wörter erfunden, sondern die geschöpften, d.h. die aus dem Abraum der Überlieferung isolierten Wortkörper werden anders ausgelesen. Sie werden anders segmentiert und eröffnen somit ihr poetisches Potential. Dichtung entsteht aus »Altablagerungen« auf Halden, Deponien, »Abraumhäfen und Novalien« (Egger 2001, 65). Es geht Egger nicht um die Entdeckung einer verborgenen poetischen Substanz, sondern um die Etablierung einer anderen Verbindung der Wörter, die weder metonymisch als Prosa noch metaphorisch als Lyrik organisiert ist. Diese liaison produziert ein neues Wissen, welches in den Wörtern das aufscheinen lässt, was in ihrer Vereinzelung nicht zur Sprache kommen kann. Dichtung stellt aus diskreten 
Wörtern kontinuierliche Stetigkeiten her: »in liaison mit allenthalben anderen (und vorigen und solchen, die noch werden wollen und selbdritt vergegenwärtigen)«. Es entsteht in der liaison der Wörter die Möglichkeit einer Verbindung zwischen Gegenwart, Vergangenheit und Zukunft. Diese sich für den Möglichkeitssinn von Sprache, für andere Bedeutungen, die nicht durch den Verweis auf eine feste Verwurzelung in einer Landschaft, dem Bereich des Eigenen und Bekannten, sondern durch eine Rasterung der Bewegung des Fremden und Unbekannten zugänglich werden. So wird die Kölner Bucht zur Homotopie des Jardin de Tuileries und die Reste einer vergangenen Industrielandschaft zum Ungrund gegenwärtiger Poesie bzw. zu einer »Georgica der Gegenwart« (Egger 2000, o. S.).

\section{Literaturverzeichnis}

Bateson, Gregory: Mind and Nature. A Necessary Unity. New York 1979.

Becker, Jürgen: Aus der Kölner Bucht. Frankfurt am Main 2009.

Benjamin, Walter: Die Aufgabe des Übersetzers. In: ders.: Gesammelte Schriften. Bd. IV,1. Hrsg. v. Rolf Tiedemann und Hermann Schweppenhäuser. Frankfurt am Main 1991, 9-21.

Brandes, Dietmar und Detlef Griese: Siedlungs- und Ruderalvegetation von Niedersachsen. Eine kritische Übersicht. Braunschweig 1991.

Celan, Paul: Sprachgitter. In: ders.: Gesammelte Werke in fünf Bänden. Hrsg. v. Beda Allemann und Stefan Reichert, Bd. 1. Frankfurt am Main 1986, 147-149.

De Felip, Eleonore de: ১Der Rede Interieurı. Zur Frage der Intra- und Intertextualität in Oswald Eggers Poem ^Herde der Reder. In: Concordia Discors vs Discordia Concors. Researches into Comparative Literature, Contrastive Linguistics, Translation and Cross-Cultural Strategies 3 (2011), 57-82.

Dietz, Josef: Die Bonner Flurnamen. Bonn 1973.

Donhauser, Michael: Waldwand. Eine Paraphrase. Berlin 2016.

Egger, Oswald: Herde der Rede. Poem. Frankfurt am Main 1999.

Egger, Oswald: Über die natürliche Magie der Einbildungskraft. Ein Briefgespräch in zehn Kapiteln mit Michael Braun. Heidelberg 2000. In: https://www.heidelberg.de/hd/HD/Rat haus/Interview+mit+Oswald+Egger.html [abgerufen am 22.7.2020].

Egger, Oswald: Nichts, das ist. Frankfurt am Main 2001.

Egger, Oswald:-broich. Homotopien eines Gedichts. Wien 2003.

Egger, Oswald: Diskrete Stetigkeit. Poesie und Mathematik. Frankfurt am Main 2008.

Egger, Oswald: Die ganze Zeit. Frankfurt am Main 2010.

Egger, Oswald: Nach dem Muster. Vom innigen Band der Begriffe symplektischer

Wortgeflechte mit Anmerkungen von Ralph M. Kaufmann zu Oswald Eggers HölderlinSchnitten. In: Paradigmenwechsel. Wandel in den Künsten und Wissenschaften. Hrsg. v. Andrea Sakoparnig, Andreas Wolfsteiner und Jürgen Bohm. Berlin, Boston 2014, 285-308.

Egger, Oswald: Harlekinsmäntel \& andere Bewandtnisse. Berlin 2017. 
Endres, Martin: Nach dem Muster. Die Paradigmatizität der Poesie. In: Paradigmenwechsel. Wandel in den Künsten und Wissenschaften. Hrsg. v. Andrea Sakoparnig, Andreas Wolfsteiner und Jürgen Bohm. Berlin, Boston 2014, 277-284.

Foucault, Michel: Andere Räume. Übers. v. Walter Seitter. In: Aisthesis. Wahrnehmung heute oder Perspektiven einer anderen Ästhetik. Hrsg. v. Karlheinz Barck, Peter Gente und Stefan Richter, Leipzig 1992, 34-46.

Grimm, Jacob und Wilhelm (Hg.): Deutsches Wörterbuch. 16 Bände in 32 Teilbänden. Leipzig 1854-1961.

Günzel, Stefan (Hg.): Topologie. Zur Raumbeschreibung in den Kultur- und Medienwissenschaften. Bielefeld 2007.

Hamacher, Werner: Parusie, Mauern. Mittelbarkeit und Zeitlichkeit, später Hölderlin. In: Hölderlin-Jahrbuch 34 (2004-2005), 93-142.

Haverkamp, Anselm: Paradigma Metapher/Metapher Paradigma. In: ders.: Die paradoxe Metapher. Frankfurt am Main 1998, 268-288.

Hunold, Alfred: Vorgermanische Ortsnamen im nördlichen Rheinland. Überlegungen zum vaskonischen, ureuropäischen Ursprung von noch heute genutzten Namen. Norderstedt 2016.

Kießling, Alois und Max Matthes (Hg): Textil-Fachwörterbuch. Berlin 1993.

Nancy, Jean-Luc: Entwurzelnde Landschaft. In: ders.: Am Grund der Bilder. Übers. v. Emmanuel Alloa. Zürich, Berlin 2006, 91-108.

Prammer, Theresia: ১Das Substrat glühtı. Oswald Egger, im Wegzusammenhang. In: Der Dichter und sein Schatten. Emphatische Intertextualität in der modernen Lyrik. Hrsg. v. Uta Degner und Elisabetta Mengolda. München 2014.

Ritter, Joachim: Landschaft. Zur Funktion des Ästhetischen in der modernen Gesellschaft. In: ders.: Subjektivität, Frankfurt am Main 1974, 141-163.

Schiffer, Peter: Führer durch das Broichtal und nächste Umgebung. Höngen 1908.

Siegert, Berhard: (Nicht) Am Ort. Zum Raster als Kulturtechnik. In: Thesis. Wissenschaftliche Zeitschrift der Bauhaus-Universität 3 (2003), 92-104.

Simons, Oliver: Nicht-euklidische Räume. In: Handbuch Literatur und Raum. Hrsg. v. Jörg Dünne und Andreas Mahler. Berlin, Boston 2015, 272-284.

Smuda, Manfred: Natur als ästhetischer Gegenstand und als Gegenstand der Ästhetik. Zur Konstitution von Landschaft. In: Landschaft. Hrsg. v. Manfred Smuda. Frankfurt am Main 1986, 44-69.

Steinmann, Holger: Sperrpoetologie. Zu Paul Celans sSprachgitter«. In: Treibhaus. Jahrbuch für die Literatur der fünfziger Jahre 5 (2009), 104-116.

Steinmann, Holger: `Vor dem Gitter hört es nicht aufı. Zur Konstituierung und Suspendierung von Innen und Außen in Franz Kafkas Betrachtung. In: Kafkas Betrachtung. Hrsg. v. Harald Neumeyer und Wilko Steffens. Würzburg 2013, 287-293.

tom Dieck, Tammo: Topologie. Berlin, New York 2000.

Voigt, Wolfgang: Bergehalden im Aachener Revier - eine Zukunft für die Natur. In: https://www.nabu-aachen-land.de/dokumentationen/haldenkonzept/ [abgerufen am 24.4. 2021].

Voigt, Wolfgang: Natur in Alsdorf - ein naturkundlicher Spaziergang. In: https://www.wolf gang-voigt.de/\%C3\%BCber-mich/ver\%C3\%B6ffentlichungen/2-broichbachtal/ [abgerufen am 25.7. 2020]. 